\title{
Satisfying the Hunger for Mobile Online Games: Providing Quality Time in Vehicular Scenarios
}

\author{
Jose Saldana \\ Communication Technologies Group (GTC) \\ University of Zaragoza \\ Zaragoza, Spain \\ jsaldana@unizar.es
}

\author{
Gustavo Marfia, Marco Roccetti \\ Department of Computer Science \\ University of Bologna \\ Bologna, Italy \\ \{marfia,roccetti\}@cs.unibo.it
}

\begin{abstract}
An increasing number of players now normally enjoy playing online games while on the move, directly from their smartphones. One barrier, however, still prevents from enjoying a fully mobile experience, as cellular network technologies do not appear capable, today, of supporting the high interactivity that the streams of some game genres (e.g., First Person Shooters) require. Our contribution is that of proposing a mechanism capable of dealing with such problem while moving in a car. Knowing in advance WiFi access point positions, we propose a mechanism that estimates the contact times in an urban setting and provides valuable advice to a player regarding which tasks it will (not) presumably be able to accomplish, during the available connectivity time. The validity of our approach is confirmed in simulation utilizing a set of vehicular traces collected on the streets of Pisa, Italy.
\end{abstract}

Keywords- vehicular communications; online games; realtime services

\section{INTRODUCTION}

Online games have entered that pool of activities that can be pursued while experiencing some spare time (e.g., reading the newspaper, solving crosswords, etc.), for example while moving from one location to another or while waiting in line. Conscious of this enormous potential (the mobile gaming market is expected to reach $\$ 54 \mathrm{~B}$ by 2015 [1]), many new mobile app titles have been published, ranging from gambling and sports to First Person Shooter (FPS) games (which typically lay down very stringent requirements in terms of interactivity). Specialized gaming sites, however, report terrible lags when accessing FPS servers from $3 \mathrm{G}$ and $4 \mathrm{G}$ access networks (a death warrant for any FPS) and suggest that better syncing should be achieved between players around the globe. This will remain a hard task until the round-trip-time experienced by communication packets over $3 \mathrm{G} / 4 \mathrm{G}$ access networks will fall between $50 \mathrm{~ms}$ to $400 \mathrm{~ms}$ on average, values that strike a hard blow to fast interactivity [2]. However, a viable way around cellular network inadequacies exists: the use of $\mathrm{WiFi}$ hotspots (i.e., they provide higher bandwidths, are cheaper and are less burdensome in terms of delays) [3].

Nevertheless, relying on WiFi APs while moving can be very challenging, if not impossible: coverage areas are typically limited, with negative consequences on any online game. Such problem becomes particularly exacerbated under vehicular mobility patterns: the duration of contact times with APs is subject to a great variability. In an urban setting, for example, no two traversals of a street require the same time, as this depends on current traffic conditions. This said, a path for enabling the use of highly interactive online games in vehicular scenarios runs through the prediction of AP-mobile device contact times. In fact, if informed that there is no available time to complete a given task, a player can avoid the frustration of remaining stuck in the middle of it. Contrarily, if sufficient time is available, a player may decide to enjoy a whole round. In essence, exploiting the prediction of how long contact times will be, a player can access only those gaming options that can be completed correctly. In this context, our contribution amounts to the design and implementation of a mechanism that may serve as a predictor of the effective contact time between a moving vehicle and an AP, i.e., the contact time for which the mean opinion score of the connection falls above an acceptable threshold. In this paper we show how, in an urban setting, that time can be typically approximated with the delay incurred on the portion of road where the AP is located. The validity of our approach is proven in simulation, where we measure the effective contact time between a moving vehicle and an AP, utilizing real mobility traces that were collected on the road, while performing over one hundred traversals of an urban street in Pisa, Italy.

\section{The Problem, Its Solution with Results}

In a very mobile environment, as one given by a vehicle moving in an urban setting, abrupt connections and disconnections can frequently occur. In fact, let us assume that a player, say Bob, wants to spend his time playing online, while moving on a car (i.e., clearly not while driving). Nevertheless, having no idea whether any WiFi connections will be available along the way, he at first spends some time searching for an available AP. Assuming he is lucky, he finds an AP and begins with a new stage of his favorite game. However, while playing, the car reaches the end of the coverage area and forcedly disconnects him. This could clearly represent a heavy disincentive for the start of any new gaming session while traveling on a car.

Now, let us imagine the experience that Bob might instead have, if a mechanism existed that knew where available APs were (e.g., open ones or APs that fell within Bob's subscription) and for how long those APs would be in touch. In such situation Bob (or a software system) would 
be able to make an informed decision and choose to play only when assured that, with a very good degree of certainty, a game that started could gracefully end, without sudden interruptions. Clearly, at times such mechanism would advise against playing, also this being a cause of disappointment, but less than that caused by a sudden game interruption. With these two situations in mind, we can see, hence, three possible outcomes of an experiment where Bob decides to play while on the move. In the best case, Bob will be able to start and end his game, thus obtaining the maximum return, in terms of enjoyment. In the worst one, Bob will access the game, but feel very bitter once he will see his tournament end because of a network disconnection. In a third situation, a mechanism might prevent Bob from playing if available gaming time resulted insufficient.

If such mechanism were always right, i.e., it always provides the right guess, Bob might risk being annoyed when prohibited to play, but would not risk an abrupt disconnection from his game. Now, a mechanism like the one described should be able to: (a) estimate the current position of the vehicle it is running on, (b) know the positions of available APs, and, (c) estimate the contact time between a vehicle and its nearest AP. While (a) and (b) can be assumed to be implementable through the use of navigation device and a database, estimating the information listed in (c) may result more challenging. Inspired by [4], [5], [6], a direction to go is that of estimating the contact time between a vehicle that is traversing a given road and a roadside AP, as the total amount of time that would be spent on it. Hence, the idea is that of approximating the contact time with a given roadside AP with the time spent on the corresponding road (i.e., portion of road between two intersections) where that AP is located. From a purely theoretical perspective, these values could be very different, as an AP might not cover the entire road section, or the connection quality, between a moving vehicle and an AP, might not be good on that road. In practice, however, relevant literature reveals that such assumption is not unpractical, considering that the average length of a street section (between two intersections) in London, for example, amounts to about $100 \mathrm{~m}$ and the typical coverage radius of an AP exceed such value. In addition, we also observe that the average speed reached at beginning of an urban road section is typically higher than that experienced on the entire road [4], [5]. This means that if we measure the time $T_{10 \%}$ that is spent by a vehicle on the initial $10 \%$ of a given urban road section, a conservative estimate (i.e., worst case scenario in terms of game playing) of the time that that vehicle will spend on the entire road section is readily computed as $10 \times T_{10 \%}$.

In order to test a contact time Prediction Mechanism (PM) based on such idea, we used the estimates computed over the first $10 \%$ and $20 \%$ of a street section, via Benedetto Croce in Pisa, where we collected real car movement traces (a total of 111 during different moments of the day and different days of the week). As the estimate that the initial subsection could produce is conservative, we introduced a factor $K>1$ that multiplied the prediction time (i.e., $T=K$ $\times(L / l) \times T_{l}$, assuming the given subsection is $l \mathrm{~m}$ long, the whole street section is $L \mathrm{~m}$ long and that the time spent on that subsection equals to $T_{l}, T$ represents the time spent on the whole road section). Columns 1 to 7 in Fig. 1 show the result of using the PM to compute $T$ (for different values of $K$ ), and put it to good use to decide whether a given game that would last $60 \mathrm{~s}$ can be played or not.

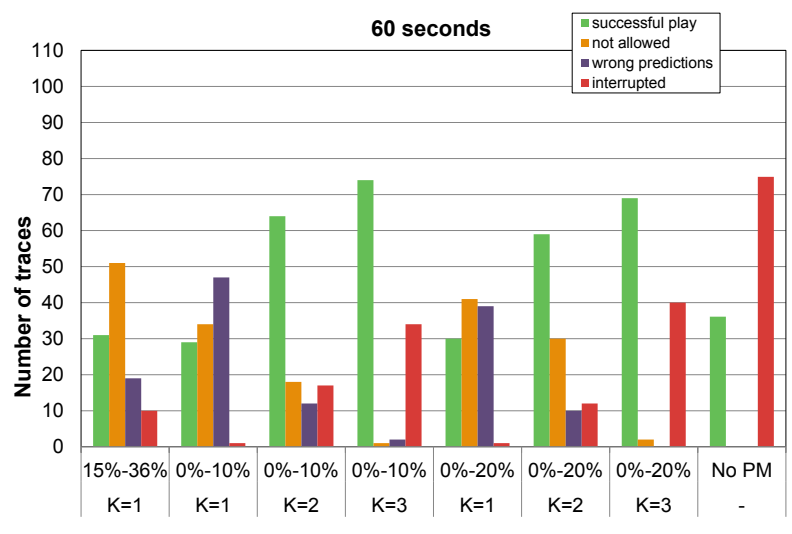

Figure 1. Results of the prediction mechanism for a $60 \mathrm{~s}$ long game.

The last column (no PM) presents the results when no prediction mechanism is used: in this case, if the player has no information concerning when a game can start and whether it would successfully end. The results, for this case, are the average over 10 tests, where the player begins the game in a uniformly distributed moment of the movement trace, just as it is in reality, when no information is known. The number of times the game ends abruptly outnumbers the number of successful endings by a factor of 2 .

\section{ACKNOWLEDGMENT}

This work has been partially financed by CPUFLIPI Project (MICINN TIN2010-17298), MBACToIP Project, of Aragon I+D Agency and Ibercaja Obra Social, NDCIPIQQoE Project of Catedra Telefonica, Univ. of Zaragoza and by the Italian PRIN ALTER-NET Project.

\section{REFERENCES}

[1] Juniper Research: Whitepaper "Entertainment on the move! Markets, Opportunities \& Forecasts 2011-2015".

[2] C. Chambers, W. Feng, S. Sahu, D. Saha, "Measurement-based Characterization of a Collection of On-line Games," Proc. 5th ACM SIGCOM conference on Internet Measurement (IMC'05). USENIX Association, Berkeley (2005).

[3] G. Marfia, M. Roccetti, "Dealing with wireless links in the era of bandwidth demanding wireless home entertainment," Proc. 2010 IEEE International Conference on Multimedia and Expo, ICME 2010, pp. 1376-1381.

[4] G. Marfia, M. Roccetti, C. E. Palazzi, A. Amoroso, "Efficient vehicle-to-pedestrian exchange of medical data: an empirical model with preliminary results," Proc. Int. Symposium on Mobile Ad Hoc Networking and Computing (MobiHoc), 2011, First ACM MobiHoc Workshop on Pervasive Wireless Healthcare, Article 3, 4 pages.

[5] G. Marfia, M. Roccetti, "Vehicular Congestion Detection and ShortTerm Forecasting: A New Model with Results". IEEE Transactions on Vehicular Technology, IEEE Vehicular Technology Society, Vol. 60, n. 7, pp. 2936-2948, 2011.

[6] J. Saldana, G. Marfia, M. Roccetti, "First Person Shooters on the Road: Leveraging on APs and Vanets for a Quality Gaming Experience," Proc. 5th IFIP/IEEE Wireless Days Conference (WD'12), IEEE, Dublin, November 2011. 